\title{
Biochemical markers and levels of physical activity related to the hepatic condition of patients infected by the hepatitis $\mathrm{C}$ virus
}

\author{
Matheus Santos de Sousa Fernandes ${ }^{1}$, Lucas de Lucena Simões e Silva ${ }^{1}$, \\ Gabriela Carvalho Jurema Santos ${ }^{4}$, Érika Rabelo Forte de Siqueira ${ }^{23}$
}

\begin{abstract}
${ }^{1}$ Superior School of Physical Education, University of Pernambuco, Recife, Pernambuco, Brazil; ${ }^{2}$ Instituto do Figado and Transplants of Pernambuco, Recife, Pernambuco, Brazil; ${ }^{3}$ Department of Gastroenterology, University of Pernambuco, Recife, Pernambuco, Brazil; ${ }^{4}$ Nutrition Department, University Federal of Pernambuco, Recife, Pernambuco, Brazil
\end{abstract}

\section{Summary}

Study aim: The study is quantitative, with a cross-sectional comparison design, and aimed to verify the association between physical activity (PA) levels and biochemical markers linked to liver function of patients infected with hepatitis $\mathrm{C}$ virus (HCV).

Material and methods: The following biochemical variables were analyzed: alanine aminotransferases (ALT), gamma-glutamyl transpeptidase $(\gamma \mathrm{Gt})$, and alkaline phosphatase (FA), these being good markers for hepatic damage, besides glycemia, HDL, LDL, and triglycerides. The International Physical Activity Questionnaire (IPAQ), short version, was used to evaluate the levels of physical activity, being classified as active and physically inactive.

Results: The sample consisted of 84 individuals aged $56.2 \pm 11.6$ years; there was a predominance of males, patients with different socioeducational patterns and those followed up for more than 6 months in the hepatology service with positive serology for HCV and HCV (RNA+), attending the Institute of Liver and Transplants of Pernambuco (IFP). The results obtained in this study indicate smaller values of the median with maximum and minimum scores of the analyzed parameters in patients considered to be physically active (ALT: $p=0.43, F A: p=0.86$ and $\gamma \mathrm{Gt}: \mathrm{p}=0.15$, glycemia: $\mathrm{p}=0.22, \mathrm{LDL}: \mathrm{p}=0.58, \mathrm{HDL}: \mathrm{P}=0.004$, triglycerides: $\mathrm{p}=0.47$ ), in comparison with the physically inactive patients. It is concluded that patients who have a higher level of physical activity present lower values of biochemical markers, positively affecting their hepatic condition.

Conclusions: There was only a significant difference in the HDL variable, but the patients with a lower level of physical activity showed greater values in general, less in the triglyceride variability. In order to test these and other important variables and improve the quality of life and health by a more direct method.

Keywords: Physical activity - Hepatology and health

\section{Introduction}

Hepatitis $\mathrm{C}$ virus (HCV) is a major public health problem worldwide, leading in many cases to liver transplants [1]. It affects $5 \%$ of the world population, which represents around 170 million people [2], and is the main causes of liver transplantation. A drug that can cure this viral infection is still not available in the pharmaceutical market [3]. However, more effective drugs including daclatasvir have led to improved management. The main pathophysiological features that normally affect the hepatic condition of patients with hepatitis $\mathrm{C}$ are related to chronic inflammation and an increase in the deposition of collagen within the hepatic extracellular matrix, which characterizes the process of fibrosis $[4,5]$. A simpler way of assessing the degree of liver damage of a patient is summarized in the continuous evaluation of the biochemical profile, the most used and important components being aspartate aminotransferase (AST), alanine aminotransferase (ALT), gamma glutamyl transpeptidase (gGt) and alkaline phosphatase (AP) [6], which are generally related to the primary signaling process for aggression within the liver of these patients affected by HCV infection [7].

Thus, studies have demonstrated that the regular practice of physical activity in several modalities has generated significant benefits in the liver [8, 9, 10], immunological [11] and hemodynamic [12], helping in a mutual 
way the treatment of those patients who have a condition of imbalance in their biochemical profile, caused not only by the virus but also by other factors such as lipogenesis, inflammation and oxidative stress. Also, it is known that the practice of regular physical activity of moderate to vigorous intensity can cause positive immune modulation, decreasing the expression of pro-inflammatory cytokines, in addition to decreasing the production of reactive oxygen species, thus gradually improving the hepatic condition of patients who are affected by these pathophysiological factors, accumulating with the progressing severity of their health condition. This promotes an evaluation of how these parameters (physical activity and biochemical profile) contribute to the better hepatic function of patients with hepatitis $\mathrm{C}[13]$.

Based on the importance of the practice of regular physical activity, there are still many gaps in knowledge related to the interaction between physical activity, biochemical profile and hepatitis C. Seeking to understand how a tool (physical activity) can be included in the treatment of patients with hepatitis $C$, the present study aimed to verify the levels of physical activity, biochemical profile and its relation with the liver function of patients infected with the hepatitis $\mathrm{C}$ virus.

\section{Materials and methods}

\section{Subjects}

The present study is quantitative, with a cross-sectional comparison. 84 patients aged $56.2 \pm 11.6$ years of both sexes, with a predominance of males, patients with different socioeducational patterns and those who are followed up for more than 6 months in the hepatology service who had positive serology for hepatitis $\mathrm{C}($ anti-HCV + ), were selected to participate in the study, with the following inclusion criteria: patients who did not have viral co-infections, attended regularly the Institute of Liver and Transplants of Pernambuco, a center of reference in care and diseases in the liver. The sample was obtained by contingent sampling within the ambulatory department of viral hepatitis of the IFP. All patients participated in the present research voluntarily, being informed of the research objectives and subsequently signing the free and informed consent form, according to the approval of this research project by the ethics and research committee, with CAAE: 48823015.3 .0000 .5192 , second resolution $466 / 12$ of studies with humans.

\section{Instruments}

In the first contact with the patient, a clinical analysis form was used to obtain sociodemographic information (name, sex, age, date of birth, origin, etc.) and clinical history information (form and time of infection, presence of diseases). In the analysis techniques we used the International Physical Activity Questionnaire, short version, which has validity and reproducibility for the Brazilian population [14], with easy-to-understand questions. For the analysis of the levels of physical activity we considered integral and fundamental elements for the practice of regular PA: frequency and duration of various activities with moderate to vigorous intensity. These are described as those that bring the best benefits to human health [15]. In order to classify physical activity levels, the World Health Organization (WHO) guideline, which defines physically active individuals, is applied to those who practice 150 minutes of moderate physical activity per week or 75 minutes of vigorous physical activity per week, distributed at least in sessions of 10 minutes [16]. Individuals who did not reach these values were considered insufficient physically active. The values of the biochemical variables ALT, AP, low density lipoprotein (LDL), high density lipoprotein (HDL), triglycerides (TG) and gGt were obtained through the clinic shelters contained in the Institute of Liver and Transplants of Pernambuco

\section{Procedures}

The questionnaires were explained in detail to the interviewed patients, being answered with objectivity, clarity and precision. If doubts arose about any question concerning the clinical or international questionnaire of physical activity, they were immediately clarified to avoid any embarrassment on the part of the evaluated subject.

\section{Statistical analysis}

Descriptive statistics were used with median values with maximum and minimum scores of each variable. After an exploratory analysis of the data, was evaluated through the D'Agostino and Person test, where it was observed that the values of the continuous variables ALT, AP, LDL, HDL, TG and $\gamma$ Gt did not meet the normality pattern. The Mann-Whitney test was used to evaluate the inter-group differences. For all the analyses, $\mathrm{p}<0.05$ was considered significant.

\section{Results}

The sample consisted of 84 patients with a mean age of 56.4 years. Among the patients there were no dropouts during the application of the clinical file and questionnaires. They were divided according to the level of physical activity into two groups with 64 physically active patients and 20 physically inactive patients. Table 1 shows the characterization of the samples as mean age and standard deviation, gender and level of physical activity.

The Mann-Whitney test did not reveal significant differences in the variables related to the hepatic condition 
Table 1. Biochemical profile, comparison between active versus inactive physical groups

\begin{tabular}{lccc}
\hline Median (min-max) & Inactive patients & Active patients & p value \\
\hline Alanine aminotransferase (U/L) & $71.5(25-452)$ & $70 *(7-487)$ & 0.4340 \\
rglutamiltranspeptidase (U/L) & $112.5(3-378)$ & $96(15-482)$ & 0.1532 \\
Glicemia (D/L) & $95.5(72-137)$ & $92(63-331)$ & 0.2272 \\
Triglycerides (mg/dL) & $100(54-172)$ & $106(47-356)$ & 0.4746 \\
HDL (mg/dL) & $39.5(21-73)$ & $42.5(28-356)$ & $<\mathbf{0 . 0 0 1}$ \\
LDL (mg/dL) & $99(46-198)$ & $94(44-193)$ & 0.5827 \\
Alkaline Phosphatase (mg/dL) & $80(50-236)$ & $83(41-363)$ & 0.8689 \\
\hline
\end{tabular}

*Values expressed in median with maximum and minimum scores and significance difference

when comparing the inter-group values: ALT: $p=0.43$, FA: $p=0.86$ and $\gamma \mathrm{Gt}: \mathrm{p}=0.15$. Glycemia: $\mathrm{p}=0.22$, LDL: $\mathrm{p}=0.58$, HDL: $\mathrm{p}=0.004$, triglycerides: $\mathrm{p}=0.47$. There was a significant difference in HDL, showing the best profile of this variable in physically active patients. In the graphical analysis, the best absolute values were found in the patients with the highest level of physical activity, which contributes to a better hepatic condition and functionality.

\section{Discussion}

The results of the present study indicate that there was heterogeneity in the number of physically active patients (64) and physically inactive patients (20). The present study demonstrated that inactive patients physically active obtained biochemical markers (LDL and glycemia) a hepatic transaminases and alkaline phosphatase in relation to the physically active ones, which can imply in a worse hepatic condition. In the insufficiently active group we can observe that the values found in the active group can be justified because the individuals in this age group are very involved with domestic and work activities that demand a higher level of intensity to be performed, even with some physiological decompensation within the menstrual cycle [16] among women, generating a significant calcium loss over the years [17]; however, because they are more physically active, they possibly present a lower burden of osteoarticular damage in relation to physically inactive women.

The early start of working life in men and those who are often not used to an active environment for them may be related to the low percentage of active men found in the present study, which has led to the prevalence of chronic degenerative diseases in men, since in addition to a low level of physical activity, they present picture of food inadequacy, in line with the findings of Bouchard et al. (1994), a classic study concerning health and physical activity. Regarding the results found in the biochemical profile found in patients with $\mathrm{HCV}$, it was visualized that the levels closer to normal were found in patients with a higher level of physical activity, in line with the study of El Kader et al. [1]. Decrease in the levels of AST, ALT, FA and $\gamma$ Gt through the effects of aerobic training within a 12 -week period of aerobic exercise. The study showed that such intervention is effective for the purpose of decreasing transaminase values. After the protocol, even patients in the studies who were within the reference values still showed a decrease in the absolute values of such variables.

Vandyck et al. [18] evaluated 17 HCV-infected individuals without drug treatment, where they investigated: anthropometric standards, metabolic status and immunological response of these patients, submitted to a walking protocol for six months. The results showed that $70.0 \%$ of them were obese or overweight and $77.0 \%$ had peripheral resistance insulin. Plasma levels of ALT were observed to decrease $(106 \pm 93 \mathrm{U} / \mathrm{L}$ vs. $59 \pm 32 \mathrm{U} / \mathrm{L}, \mathrm{p}<0.01)$, in the ALT / AST rate $(1.04$ vs. 0.70, p <0.01), in triglycerides $(165 \pm 86 \mathrm{mg} / \mathrm{dL}$ vs. $124 \pm 49 \mathrm{mg} / \mathrm{dL})$ and peripheral resistance to insulin (4.0 vs. 2.7 ).

In addition, $88.0 \%$ of the individuals felt better health at the end of the six-month protocol. Another extremely relevant result of this study was the reduction in viral load detected in four individuals. This is due to modulation of the exercise [4]. Some limitations can be seen in this study, including the way of verifying the levels of physical activity through IPAQ, short version. Even though it is a viable tool, it is an indirect way to evaluate this variability more reliably in the different social domains (leisure, sport, etc.).

In addition, despite the significance of biochemical variables and the demonstration of an association between physical activity, biochemical profile and better hepatic function of patients with $\mathrm{C}$ virus, a larger sample could result in greater external validity. In summary, the present study showed that regular physical activity levels in patients with hepatitis $\mathrm{C}$ can improve the values of the 
biochemical profile of these patients, resulting in a better hepatic condition, due to the reduction of the hepatic impairment.

\section{Conclusion}

There was only a significant difference in the HDL variable, but the patients with a lower level of physical activity showed greater values in general, less in the triglyceride variability. In order to test these and other important variables and improve the quality of life and health by a more direct method.

\section{Conflict of interest: Authors state no conflict of interest.}

\section{References}

1. Abd El-Kader S.M., Al-Jiffri O.H., Al-Shreef F.M. (2014) Liver enzymes and psychological well-being response to aerobic exercise training in patients with chronic hepatitis C. Afr. Health Sci., 14(2): 414-419. DOI: 10.4314/ahs. v14i2.18.

2. Arthur M.J. (2000) Fibrogenesis II. Metalloproteinases and their inhibitors in liver fibrosis. Am. J. Physiol. Gastrointest. Liver Physiol., 279: G245-G249.

3. Bataller R. Brenner D.A. (2005) Liver fibrosis. The Journal of Clinical Investigation, Volume 115 Number 2 February. DOI: 10.1172/JCI24282.

4. Berzigotti A., Saran U. Dufour J.F. (2016) Physical Activity and liver diseases; Hepatology, 63(3): 1026-1040. DOI:10.1002/hep.28132.

5. Duffield J.S. Forbes S.J., Constandinou C.M., Clay S., Partolina M., Vuthoori S., Wu S., Lang R., Iredale J.P. (2005) Selective depletion of macrophages reveals distinct, opposing roles during liver injury and repair. $J$. Clin. Invest., 115: 56-65; DOI: 10.1172/JCI22675.

6. El-Serag H.B. (2012) Epidemiology of viral hepatitis and hepatocellular carcinoma. Gastroenterology, 142(6): 1264-1273.

7. Fallowfield J.A., Mizuno M., Kendall T.J., Constandinou C.M., Benyon R.C., Duffield J.S., Iredale J.P. (2007) Scar-associated macrophages are a major source of hepatic matrix metalloproteinase-13 and facilitate the resolution of murine hepatic fibrosis. J. Immunol., 178: 52885295. PMID: 17404313.

8. Friedman S.L. (2008) Hepatic stellate cells: protean, multifunctional, and enigmatic cells of the liver.
Physiol Rev., [S.1], v. 88, n. 1, p. 125-72. DOI: 10.1152/ physrev.00013.2007.

9. Gonçalves I., Oliveira P. Ascensão A. Magalhaes J. (2013) Exercise as a therapeutic tool to prevent mitochondrial degeneration in nonalcoholic steatohepatitis; Eur. J. Clin. Invest., 43(11): 1184-1194. DOI: 10.1111/eci.12146.

10. Holt M.P., Cheng L. Ju C. (2008) Identification and characterization of infiltrating macrophages in acetaminophen-induced liver injury. J. Leukoc. Biol., 84: 1410-1421. DOI: 10.1189/jlb.0308173.

11. Lavanchy D. (2009) The global burden of hepatitis C. Liver Int., 29(s1): 74-81. DOI: 10.1111/j.1478-3231 .2008.01934.x.

12. Matsudo V.K.R., Matsudo S.M.M. (2000) Evidencias da importância da atividade física nas doenças cardiovasculares e na same. Diagnostico \& Tratamento, v.5, n.2, p. 10-17.

13. Mehal W.Z., Iredale J., Friedman S.L. (2011) Scraping fibrosis: expressway to the core of fibrosis. Nature Med., 17: 552-553. DOI: 10.1038/nm0511-552.

14. Nasif S., Halawa F., Fouad A., Abdel Hafez H., Kamel A., Rezk Allah S. (2009) Effect of Aerobic Exercise on Patients with Chronic Non-Cirrhotic Active Hepatitis C Virus (HCV). Med. J. Cairo Univ., 77: 81-87.

15. Pellicoro A., Ramachandran P., Iredale J.P., Fallowfield J.A. (2014) Liver fibrosis and repair: immune regulation of wound healing in a solid organ, Nature Reviews Immunology. DOI: 10.1038/NRI3623.

16. Strauss E. (2001) Hepatite C. Revista da Sociedade Brasileira de Medicina Tropical, 34(1): 69-82.

17. Oh S., Shida T., Yamagishi K., Tanaka K., So R., Tsujimoto T., Shoda J. (2015) Moderate to vigorous physical activity volume is an important factor for managing nonalcoholic fatty liver disease: A retrospective study. Hepatology, 61(4): 1205-1215. DOI: 10.1002/hep.27544.

18. Rusu E., Jinga M., Enache G., Rusu F., Dragomir A.D., Ancuta I., Draguț R., Parpala C., Nan R., Sima I., Ateia S., Stoica V., Cheța D.M., Radulian G. (2013) Effects of lifestyle changes including specific dietary intervention and physical activity in the management of patients with chronic hepatitis $\mathrm{C}$ - a randomized trial, Nutr. J., 12(1): 119.

\section{Received 25.07.2017 \\ Accepted 10.01.2018}

(C) University of Physical Education, Warsaw, Poland 